\title{
ADAPTED INTEGRAL REPRESENTATIONS BY MEASURES ON CHOQUET BOUNDARIES
}

\author{
BY DIEDERICH HINRICHSEN
}

Communicated by V. Klee, March 31, 1966

1. Introduction. In recent years, the following abstract CauchyWeil Theorem has been established by different methods [8], [7], [4]:

Let $X$ be a relatively compact domain in $C^{n}$ or more generally in a separable analytic space $(Y, \mathcal{O})$. Let $H$ be the algebra of continuous complex valued functions on $\bar{X}$ which are holomorphic in $X$. Then there exists a family $\left(\mu_{x}\right)_{x \in X}$ of complex measures on the Silov boundary $S$ of $\bar{X}$ with respect to $H$ such that:

(i) $\mu_{x}(f)=f(x)$ for all $f \in H, x \in X$.

(ii) $x \rightarrow \mu_{x}(g)$ is holomorphic on $X$ for all continuous complex valued functions $g$ on $S$.

$H$. Bauer [1] has raised the problem whether it is possible to concentrate the measures $\mu_{x}$ on the Choquet boundary $X_{e}(H)$ of $\bar{X}$ with respect to $H . X_{e}(H)$ is defined (for all separating vector spaces $H$ of scalar valued functions on a compact space $\bar{X}$ ) as the set of all $x \in \bar{X}$ such that the unit point measure $\epsilon_{x}$ at $x$ is the only positive Radon measure $\mu$ of total mass 1 on $\bar{X}$ satisfying $\mu(h)=h(x)$ for all $h \in H$ [6]. If $\bar{X}$ is metrizable and $H$ is a Banach algebra containing the constants $X_{e}(H)$ coincides with the minimal boundary of $H$ as defined in [3]. One can prove that for an algebra $H$ the following statements are equivalent: (a) $x \in X_{e}(H)$; (b) if $\mu(h)=h(x)$ for all $h \in H$, then $\mu(\{x\}) \neq 0$ for all complex measures $\mu$ on $\bar{X}$; (c) $\epsilon_{x}$ is orthogonal to all complex measures $\mu$ on $\bar{X}$ with $\mu(h)=0$ for all $h \in H$.

The purpose of the present paper is to give a solution of Bauer's problem by treating even the following more abstract situation:

Let $X$ be a relatively compact open subset of a Hausdorff space, $\mathfrak{e}(\bar{X}, K)$ the algebra of all continuous $K$-valued functions on $\bar{X}$ $(K=C$ or $K=R)$ and $H$ a separating linear subspace of $\mathfrak{e}(\bar{X}, K)$ with $1 \in H$. Suppose $E$ to be a vector space of $K$-valued functions on $X$ which contains the restrictions $\operatorname{res}_{X} h$ of all $h \in H$ to $X$-under which conditions is there a family $\left(\nu_{x}\right)_{x \in X}$ of ( $K$-valued) Radon measures concentrated on the Choquet boundary $X_{e}=X_{e}(H)$ such that:

(i) $\left(\nu_{x}\right)$ is a family of $H$-representing measures, i.e. $\nu_{x}(h)=h(x)$ for all $h \in H, x \in X$;

(ii) $\left(\nu_{x}\right)$ is $E$-adapted, i.e. $x \rightarrow \nu_{x}(g)$ is in $E$ whenever $g \in \mathfrak{e}\left(\bar{X}_{e}, K\right)$ ? [4].

Important suggestions for our solution were given by $\mathrm{L}$. Bungart 
I would like to express my gratitude to Professor Heinz Bauer who supported my research in many ways by his advice.

Details, extensions and further applications will appear elsewhere.

2. Preliminaries. All topological vector spaces we consider are Hausdorff spaces. If $E$ and $F$ are locally convex spaces, we denote by $E \widehat{\otimes} F$ the completion of the projective topological tensor product of $E$ and $F$. We introduce the following terminology:

DeFINITION 1. Let $E$ be a vector space of scalar valued functions on a set $T$ and $F$ a topological vector space. Then the vector space $E(T, F)$ of all functions $f: T \rightarrow F$ for which $t \rightarrow\left\langle f(t), y^{\prime}\right\rangle$ lies in $E$ whenever $y^{\prime} \in F^{\prime}$, is called the vector space of E-morphic mappings from $T$ into $F$.

The following theorem of A. Grothendieck yields the basis for all applications of his results in [9] to function theory:

THEOREM 2.1. Let E be a nuclear \&F-space of scalar valued functions on a set $T$. Then, for any complete locally convex space $F, E \widehat{\otimes} F$ is canonically isomorphic to the vector space $E(T, F)$.

REMARK. Let $E$ be a nuclear $\mathcal{L F}$-space of scalar valued functions on a set $T$ and let $F_{1}, F_{2}$ be complete locally convex spaces. If $p$ is a continuous linear $F_{2}$-valued function on $F_{1}$, then the linear mapping from $E\left(T, F_{1}\right)$ in to $E\left(T, F_{2}\right)$, induced by $1_{E} \widehat{\otimes} p$ according to Theorem 2.1, maps $f \in E\left(T, F_{1}\right)$ into $p \circ f$.

The following lemma is a corollary of a well known proposition on metrizable groups:

Lemma 2.2. Let $u_{i}: E_{i} \rightarrow F_{i}$ be surjective homomorphisms from the metrizable topological vector spaces $E_{i}$ onto the topological vector spaces $F_{i}(i=1,2)$. Then $u_{1} \widehat{\otimes} u_{2}: E_{1} \widehat{\otimes} E_{2} \rightarrow F_{1} \widehat{\otimes} F_{2}$ is surjective.

3. General theorems. We return now to the abstract problem as fixed in the introduction. We denote by $\operatorname{Mr}(\bar{X})$ the Banach space of $K$-valued Radon measures on $\bar{X}$ and by $M$ the subspace of measures "concentrated on the Choquet boundary $X_{e}$ of $H$ " in the sense that they are concentrated on every border defined by functions $f \in \mathfrak{C}(\bar{X}, R)$ with respect to $\operatorname{Re}(H)$. (If $\bar{X}$ is metrizable $X_{e}$ is a $G_{\delta}$ and $M$ consists of all $\mu$ concentrated on $X_{e}$ in the ordinary sense [6]). Let $i$ be the injection of $M$ into $\Re(\bar{X})$ and let $\varphi$ be the canonical homomorphism from $\operatorname{mr}(\bar{X})$ onto $N=\operatorname{\Re r}(\bar{X}) / M_{0}$ where $M_{0}=\{\mu \in \mathfrak{M}(\bar{X}): \mu(h)=0$ for all $h \in H\}$. Then $p=\varphi \circ i$ is a continuous linear mapping from the Banach space $M$ into the Banach space $N$. By using Choquet's Existence Theorem we can prove

THEOREM 3.1. $p$ is a surjective homomorphism. 
DEFINITION 2. Let $H, E$ be two function spaces as considered in the introduction. The pair $(H, E)$ is called admissible, if the mapping $x \rightarrow \varphi\left(\epsilon_{x}\right)$ from $X$ into $N$ is an E-morphic function.

Proposition 3.2. If $(H, E)$ is admissible then, for every separating linear subspace $\tilde{H}$ of $H$ with $1 \in \widetilde{H},(\widetilde{H}, E)$ is admissible.

For the rest of this paper we suppose $E$ to be a locally convex space endowed with a topology $J$ stronger than the topology of simple convergence. $J_{k}$ will denote the topology of uniform convergence on compact sets in $X$.

Proposition 3.3. The pair $(H, E)$ is admissible if it has the following two properties:

(1) $E$ is quasicomplete and $\Im \prec J_{k}$.

(2) The unit ball of $H$ with respect to the uniform norm is equicontinuous on $X$.

Proposition 3.4. If the unit ball of $H$ is, after restriction to $X$, relatively compact in $E$ and $J \succ J_{k}$, then the pair $(H, E)$ is admissible.

CoRollary 3.5. Let $(H, E)$ be a pair such that $E$ is quasi-complete and nuclear. If $J$ is stronger than $J_{k}$ and weaker than the topology of uniform convergence on $X$, then $(H, E)$ is admissible.

The solution of our general problem is given by:

THEOREM 3.6. Let E be a nuclear Fréchet space of scalar valued functions on $X$ such that the pair $(H, E)$ is admissible. Then there exists an E-morphic $M$-valued function $x \rightarrow \nu_{x}$ on $X$ such that $\nu_{x}(h)=h(x)$ for all $h \in H, x \in X$.

REMARK. Theorem 3.6, under its assumptions, yields even more than was demanded in the introduction: The family $\left(\nu_{x}\right)$ has the property that $x \rightarrow \nu_{x}(g)$ lies in $E$ for all bounded measurable functions $g$ on $\bar{X}_{e}$.

4. Applications. (a) Let $X$ be a relatively compact domain in a harmonic space $(Y, \mathfrak{F})$ in the sense of $\mathrm{H}$. Bauer's axiomatic theory of harmonic functions [2]. Take $E$ as the (real) vector space of harmonic functions on $X$ endowed with the topology of uniform convergence on compact sets.

Proposition 4.1. $E$ is a nuclear Fréchet space.

Define $H=\left\{h \in \mathcal{C}(\bar{X}, R): \operatorname{res}_{X} h \in E\right\}$. Then we have according to Corollary 3.5: There exist real measures $\nu_{x}$ on $X^{*}$ concentrated on the set $X_{e}$ of regular points of $\bar{X}$ such that:

(1) $\nu_{x}(h)=h(x)$ for all $h \in H, x \in X$. 
(2) $x \rightarrow \nu_{x}(g)$ is harmonic on $X$ for all bounded measurable real functions $g$ on $X^{*}$.

REMARK. If $\bar{X}$ is a $H$-simplex (see [1]) the measures $\nu_{x}$ are uniquely determined and positive. The harmonic measures on $X^{*}$ constructed by the method of Perron-Wiener satisfy the conditions (1) and (2) but are in general (e.g. for the heat equation) not concentrated on $X_{e}$.

(b) Let $X$ be a separable relatively compact domain in an alytic space $(Y, \mathcal{O})$ and let $E$ be the (complex) algebra of holomorphic functions on $X$ endowed with the topology of uniform convergence on compact sets. Define $H=\left\{h \in \mathcal{e}(\bar{X}, C):\right.$ res $\left._{X} h \in E\right\}$. L. Bungart [4] proves that $E$ is a nuclear Fréchet space. Hence, by Corollary 3.5, Theorem 3.6 is applicable and yields a solution of Bauer's problem. By Proposition 3.2 this problem can be solved for all separating linear subspaces $\tilde{H}$ of $H$ with $1 \in \tilde{H}$.

REMARK. Under additional assumptions on $H$ which are verified in the case of analytic and in the case of harmonic functions, one can prove a generalization of Bungart's Theorems $B^{p}(1 \leqq p \leqq \infty)$ and $C^{2}[5]$ such that the measure $\sigma$ occurring in the integral formula is concentrated on the Choquet boundary with respect to $H$.

Added in proof. (a) The assumption that $H$ (resp. $\tilde{H}$ ) contains the constants may be omitted in our statements.

(b) The Theorem 2.1 of A. Grothendieck is cited incompletely. Here as in the following Remark, $E$ is supposed to be complete and endowed with a topology stronger than the topology of simple convergence.

\section{BIBLIOGRAPHY}

1. H. Bauer, Aspects of linearity in the theory of function algebras, Proceedings of a Symposium on Function Algebras, New Orleans (1965). (to appear)

2. - Harmonische Räume und ihre Potentialtheorie, Lecture Notes in Mathematics, Springer-Verlag, Berlin. (to appear)

3. E. Bishop, A minimal boundary for function algebras, Pacific J. Math. 9 (1959), 629-642.

4. L. Bungart, Holomorphic functions with values in locally convex spaces and applications to integral formulas, Trans. Amer. Math. Soc. 111 (1964), 317-344.

5. - Cauchy integral formulas and boundary kernel functions in several complex variables, Proc. Conf. Complex Analysis (1964), Minneapolis, 1965, pp. 7-18.

6. G. Choquet and P. A. Meyer, Existence et unicité des représentations intégrales dans les convexes compacts quelconques, Ann. Inst. Fourier 13 (1963), 139-154.

7. O. Forster, Funktionswerte als Randintegrale in komplexen Raumen, Math. Ann. 150 (1963), 317-324.

8. A. M. Gleason, The abstract theorem of Cauchy-Weil, Pacific J. Math. 12 (1962), 511-525.

9. A. Grothendieck, Produits tensoriels topologiques et espaces nucléaires, Mem. Amer. Math. Soc., Providence, R. I., 1955, 140 pp.

Mathematisches Institut Der Universität Erlangen-NÜrnberg, Germany 